\title{
On lifting invariant probability measures
}

by

\author{
Tomasz CIEŚLA
}

Presented by the Editors

Summary. We study when an invariant probability measure lifts to an invariant measure. Consider a standard Borel space $X$, a Borel probability measure $\mu$ on $X$, a Borel map $T: X \rightarrow X$ preserving $\mu$, a Polish space $Y$, a continuous map $S: Y \rightarrow Y$, and a Borel surjection $p: Y \rightarrow X$ with $p \circ S=T \circ p$. We prove that if the fibers of $p$ are compact then $\mu$ lifts to an $S$-invariant measure on $Y$.

1. Introduction. In this note we address the following question asked by Feliks Przytycki:

Question. Let $X$ be a compact metric space and $Y$ a Polish space. Let $T: X \rightarrow X$ and $S: Y \rightarrow Y$ be continuous maps. Let $p: Y \rightarrow X$ be a Borel surjection with $p \circ S=T \circ p$. Let $\mu$ be a T-invariant Borel probability measure on $X$. When does $\mu$ lift to an $S$-invariant Borel probability measure on $Y$ ?

The answer is affirmative under the assumption that the fibers of $p$ are finite and the sets $\left\{x \in X:\left|p^{-1}(x)\right|=n\right\}$ are $T$-invariant (for instance, this holds if $S$ and $T$ are homeomorphisms). A special case of this $\left(\left|p^{-1}(x)\right| \leq 2\right.$ for all $x \in X$ ) appeared in the proof of [Prz, Corollary 10.2]. An obvious modification of Przytycki's argument shows that one can lift $\mu$ to an $S$ invariant measure $\nu$ where $\nu$ is defined by

$$
\nu(A)=\int_{X} \frac{\left|A \cap p^{-1}(x)\right|}{\left|p^{-1}(x)\right|} \mathrm{d} \mu(x) .
$$

It is also known that if $Y$ is compact and $p$ is continuous then $\mu$ lifts to an $S$-invariant measure $\nu$. Note that $p$ induces the push-forward map

2020 Mathematics Subject Classification: Primary 37L40.

Key words and phrases: invariant measures, lift.

Received 25 February 2020; revised 4 March 2020.

Published online 6 April 2020. 
$p_{*}: P(Y) \rightarrow P(X)$ between the spaces of Borel probability measures which is a continuous surjection, so the preimage of $\mu$ is a non-empty compact subset $K$ of $P(Y)$. Clearly, $K$ is convex. Since $\mu$ is $T$-invariant and $p \circ S=T \circ p$, we obtain $S_{*}(K) \subset K$. Hence by Schauder's fixed-point theorem there exists $\nu \in K$ with $\nu=S_{*}(\nu)$. This means that $\nu$ is a lift of $\mu$ which is $S$-invariant.

On the other hand, if the assumption on compactness of the fibers of $p$ is dropped then it may happen that $\mu$ does not lift to an $S$-invariant measure even if $Y$ is compact, $T$ is the identity map and $S$ is a homeomorphism. For instance, let $X=\{0,1\}$ and $Y=\mathbb{Z} \cup\{\infty\}$ be the one-point compactification of the countable discrete space $\mathbb{Z}$. Let $T=\mathrm{id}_{X}, S(n)=n+1$ for $n \in \mathbb{Z}$, $S(\infty)=\infty, p(n)=0$ for $n \in \mathbb{Z}, p(\infty)=1$, and $\mu=\frac{1}{2} \delta_{0}+\frac{1}{2} \delta_{1}$. Suppose that $\nu$ is an $S$-invariant measure on $Y$. By $S$-invariance, $\nu(\{n\})=\nu(\{0\})$ for all $n \in \mathbb{Z}$. If $\nu(\{0\})=0$ then $\nu(\mathbb{Z})=\sum_{n \in \mathbb{Z}} \nu(\{n\})=0$, and if $\nu(\{0\})>0$ then $\nu(\mathbb{Z})=\sum_{n \in \mathbb{Z}} \nu(\{n\})=\infty$. In both cases $\nu(\mathbb{Z}) \neq 1 / 2$, hence $\mu$ does not lift to an $S$-invariant measure.

We shall work in a more general context. We drop the assumption on compactness of $X$ and continuity of $T$. The following result generalizes both special cases discussed above.

TheOrem 1. Let $X$ be a standard Borel space with a Borel probability measure $\mu$ and let $T: X \rightarrow X$ be a $\mu$-measurable map preserving $\mu$. Let $Y$ be a Polish space and let $S: Y \rightarrow Y$ be a continuous map. Let $p: Y \rightarrow X$ be a Borel map such that $p \circ S=T \circ p$ and $\mu(p(Y))=1$. Suppose that for $\mu$-a.a. $x \in X$ the set $p^{-1}(x)$ is compact. Then there exists a Borel probability measure $\nu$ on $Y$ which is $S$-invariant and $p_{*}(\nu)=\mu$.

One can prove an even more general result: instead of single maps $S$ and $T$ one can work with a left amenable semigroup $\Gamma$ (for instance, an abelian semigroup) acting on $Y$ by continuous maps and acting on $X$ by measurepreserving maps so that the actions of $\Gamma$ on $Y$ and $X$ commute with $p$.

Theorem 2. Let $X$ be a standard Borel space with a Borel probability measure $\mu$. Let $Y$ be a Polish space. Let $p: Y \rightarrow X$ be a Borel map with $\mu(p(Y))=1$ and such that the set $p^{-1}(x)$ is compact for $\mu$-a.a. $x \in X$. Let $\Gamma$ be a left amenable semigroup. Consider actions $\Gamma \curvearrowright Y, \Gamma \curvearrowright X$ such that:

- $\Gamma$ acts on $Y$ by continuous maps, i.e. for all $\gamma \in \Gamma$ the map $S_{\gamma}: Y \rightarrow Y$, $S_{\gamma}(y)=\gamma y$, is continuous,

- $\mu$ is $\Gamma$-invariant, i.e. for all $\gamma \in \Gamma$ the map $T_{\gamma}: X \rightarrow X, T_{\gamma}(x)=\gamma x$, preserves $\mu$,

- the actions of $\Gamma$ on $Y$ and $X$ commute with $p$, i.e. $p \circ S_{\gamma}=T_{\gamma} \circ p$ for all $\gamma \in \Gamma$.

Then there exists a $\Gamma$-invariant Borel probability measure $\nu$ on $Y$ such that $p_{*}(\nu)=\mu$. 
Clearly, Theorem 1 is a special case of Theorem 2, to see this just take $\Gamma=(\mathbb{N},+)$ with actions on $X$ and $Y$ given by $\mathbb{N} \times X \ni(n, x) \mapsto T^{n} x \in X$ and $\mathbb{N} \times Y \ni(n, y) \mapsto S^{n} y \in Y$. Therefore it is enough to prove Theorem 2 . Nevertheless, we provide a separate proof of Theorem 1 which avoids using tools from the theory of amenable semigroups.

2. Preliminaries. In this section we recall some definitions and useful facts.

A standard Borel space is an uncountable set $X$ with a $\sigma$-algebra $\Sigma$ of subsets of $X$ such that there exists a Polish (i.e. separable, completely metrizable) topology $\tau$ on $X$ whose Borel $\sigma$-algebra is $\Sigma$.

Given a topological space $Y$ we denote by $K(Y)$ the collection of all compact subsets of $Y$. The set $K(Y)$ can be endowed with the Vietoris topology, generated by all sets of the form

$$
\{K \in K(Y): K \cap U \neq \emptyset\} \quad \text { and } \quad\{K \in K(Y): K \subset U\}
$$

where $U \subset Y$ is open. If $Y$ is Polish or compact, then $K(Y)$ is Polish or compact, respectively.

For a Polish space $Y$ we denote by $P(Y)$ the set of all Borel probability measures on $Y$ endowed with the weak* topology, generated by all sets of the form

$$
\left\{\sigma \in P(Y):\left|\int_{Y} f \mathrm{~d} \sigma-\int_{Y} f \mathrm{~d} \sigma_{0}\right|<\varepsilon\right\}
$$

where $\sigma_{0} \in P(Y), f: Y \rightarrow \mathbb{R}$ is continuous and bounded, and $\varepsilon>0$. Traditionally, a somewhat erroneous terminology is in use: a sequence of measures convergent in the weak* topology is sometimes said to converge weakly. If $Y$ is a compact metric space then so is $P(Y)$.

A semigroup $\Gamma$ is called left amenable if there exists a left invariant mean for $\Gamma$ (for more details we refer the reader to [Pat, 0.18]).

3. Proofs of Theorems 1 and 2, We start with the following key lemma.

Lemma 1. Let $X$ be a standard Borel space with a Borel probability measure $\mu$. Let $Y$ be a Polish space. Let $p: Y \rightarrow X$ be a Borel map such that $\mu(p(Y))=1$. Let $M \subset P(Y)$ be the set of all measures $\sigma$ with $p_{*}(\sigma)=\mu$. If for $\mu$-a.a. $x \in X$ the set $p^{-1}(x)$ is compact then $M$ is a non-empty convex compact subset of $P(Y)$.

Proof. Suppose additionally that $Y$ is compact. The general case will be considered later.

First of all, the set $M$ is non-empty. For instance, by $\mathrm{Kec}, 18.3$ there exists a $\mu$-measurable function $u: p(Y) \rightarrow Y$ with $u(x) \in p^{-1}(x)$ for all 
$x \in p(Y)$. Define a measure $\sigma \in P(Y)$ by $\sigma(B)=\int_{p(Y)} \delta_{u(x)}(B) \mathrm{d} \mu(x)$. Then $\sigma \in M$. Secondly, it is clear that $M$ is convex. It remains to prove that $M$ is compact. Let $\nu_{1}, \nu_{2}, \ldots$ be a sequence of elements of $M$ converging to some $\nu \in P(Y)$. We shall prove that $\nu \in M$, i.e. $p_{*}(\nu)=\mu$.

Claim 1. Let $A \subset X$ be a Borel set. Then $p_{*}(\nu)(A) \geq \mu(A)$.

Proof of Claim 1. This is trivial if $\mu(A)=0$, so assume that $\mu(A)>0$. Fix $\varepsilon>0$. Endow $X$ with a Polish topology including the given Borel structure. Let $X^{\prime} \subset X$ be a Borel set of full measure such that $p^{-1}(x)$ is compact for all $x \in X^{\prime}$.

Let $f: X^{\prime} \rightarrow K(Y)$ be given by $f(x)=p^{-1}(x)$. We shall prove that $f$ is Borel. Recall that the Borel structure of $K(Y)$ is generated by all sets of the form $B=\{K \in K(Y): K \cap U \neq \emptyset\}$ where $U \subset Y$ is open (see [Kec, 12.C]). Therefore it is enough to prove that $f^{-1}(B)$ is Borel whenever $B$ is of the aforementioned form. Note that

$$
\begin{aligned}
f^{-1}(B) & =\left\{x \in X^{\prime}: f(x) \in B\right\}=\left\{x \in X^{\prime}: f(x) \cap U \neq \emptyset\right\} \\
& =\left\{x \in X^{\prime}: \exists y \in U p(y)=x\right\}=\pi_{X}\left(\operatorname{graph}(p) \cap\left(U \times X^{\prime}\right)\right),
\end{aligned}
$$

which is Borel by [Kec, 28.8]. Hence $f$ is Borel.

By Lusin's theorem there exists a non-empty compact subset $K \subset A \cap X^{\prime}$ such that $\mu(K)>\mu(A)-\varepsilon$ and $\left.f\right|_{K}: K \rightarrow K(Y)$ is continuous. Then $\{f(x): x \in K\}$ is compact in $K(Y)$, as a continuous image of a compact set. By [Kec, 4.29], $f(K)=\bigcup\{f(x): x \in K\}=p^{-1}(K)$ is compact in $Y$.

Since $\nu_{n}$ converges to $\nu$ weakly and $p^{-1}(K)$ is compact, by the Portmanteau lemma we have

$$
p_{*}(\nu)(K)=\nu\left(p^{-1}(K)\right) \geq \limsup _{n \rightarrow \infty} \nu_{n}\left(p^{-1}(K)\right)=\limsup _{n \rightarrow \infty} \mu(K)=\mu(K) .
$$

It follows that $p_{*}(\nu)(A) \geq p_{*}(\nu)(K) \geq \mu(K) \geq \mu(A)-\varepsilon$. Since $\varepsilon>0$ can be chosen arbitrarily, the claim follows.

Claim 2. Let $A \subset X$ be a Borel set. Then $p_{*}(\nu)(A) \leq \mu(A)$.

Proof of Claim 2. Claim 1 for $X \backslash A$ gives $p_{*}(\nu)(X \backslash A) \geq \mu(X \backslash A)$. This can be rewritten as $1-p_{*}(\nu)(A) \geq 1-\mu(A)$, hence $p_{*}(\nu)(A) \leq \mu(A)$.

Claims 1 and 2 imply that $p_{*}(\nu)(A)=\mu(A)$ for all Borel sets $A \subset X$. Therefore $p_{*}(\nu)=\mu$, which proves that $M$ is closed in $P(Y)$ and hence compact. This finishes the proof for $Y$ compact.

It remains to consider the case when $Y$ is non-compact. Recall that any Polish space embeds homeomorphically into the Hilbert cube $[0,1]^{\mathbb{N}}$ as a $G_{\delta}$ subset. Write $Y^{\prime}=[0,1]^{\mathbb{N}}$ for brevity and view $Y$ as a subspace of $Y^{\prime}$. Let $\Sigma$ be the Borel $\sigma$-algebra of $X$. Let $X^{\prime}=X \cup\{*\}$ and $\Sigma^{\prime}=\Sigma \cup\{A \cup\{*\}$ : $A \in \Sigma$. Then $\Sigma^{\prime}$ gives $X^{\prime}$ the structure of a standard Borel space. Let $\mu^{\prime}$ be the Borel probability measure on $X^{\prime}$ given by $\mu^{\prime}(B)=\mu(B \cap X)$ for any 
$B \in \Sigma^{\prime}$, and let $p^{\prime}: Y^{\prime} \rightarrow X^{\prime}$ be given by $p^{\prime}(y)=p(y)$ if $y \in Y$ and $p^{\prime}(y)=*$ otherwise. Note that $p^{\prime}$ is Borel. Let $M^{\prime} \subset P\left(Y^{\prime}\right)$ be the set of all measures $\sigma^{\prime}$ with $p_{*}\left(\sigma^{\prime}\right)=\mu^{\prime}$. Then $X^{\prime}, Y^{\prime}, \mu^{\prime}, p^{\prime}$, and $M^{\prime}$ satisfy the hypotheses of the lemma and $Y^{\prime}$ is compact, so $M^{\prime}$ is a non-empty convex subset of $P\left(Y^{\prime}\right)$. It is clear that the map $M \ni \sigma \mapsto \sigma^{\prime} \in P\left(Y^{\prime}\right)$ given by $\sigma^{\prime}(B)=\sigma(B \cap Y)$ maps $M$ onto $M^{\prime}$ homeomorphically. Therefore $M$ is a non-empty compact subset of $P(Y)$, which is obviously convex as well.

We prove Theorem 1 using the averaging trick.

Proof of Theorem 1. Let $M \subset P(Y)$ be the set of all measures $\sigma$ with $p_{*}(\sigma)=\mu$. By Lemma 1, $M$ is non-empty, convex and compact.

Pick an arbitrary $\sigma \in M$. For all positive integers $n$ define

$$
\nu_{n}=\frac{1}{n} \sum_{i=0}^{n-1}\left(S^{i}\right)_{*}(\sigma) .
$$

Note that for all $i$,

$$
p_{*}\left(\left(S^{i}\right)_{*}(\sigma)\right)=\left(p \circ S^{i}\right)_{*}(\sigma)=\left(T^{i} \circ p\right)_{*}(\sigma)=\left(T^{i}\right)_{*}\left(p_{*}(\sigma)\right)=\left(T^{i}\right)_{*}(\mu)=\mu,
$$

so $\left(S^{i}\right)_{*}(\sigma) \in M$ for all $i$, and since $M$ is convex we have $\nu_{n} \in M$ for all $n$. So, by compactness of $M$ there exists a subsequence $\nu_{n_{1}}, \nu_{n_{2}}, \ldots$ converging to some $\nu \in M$. Then $\nu$ is $S$-invariant by the proof of the Bogolyubov-Krylov theorem (see [Sin, Theorem 1.1]). Hence $\nu$ is as required.

The averaging trick can be used to prove Theorem 2 provided $\Gamma$ admits a Følner sequence, i.e. an increasing sequence of finite sets $F_{n} \subset \Gamma$ such that $\Gamma=\bigcup_{n \in \mathbb{N}} F_{n}$ and $\lim _{n \rightarrow \infty}\left|g F_{n} \triangle F_{n}\right| /\left|F_{n}\right|=0$ for all $g \in \Gamma$. This is the case for instance for amenable groups and for abelian semigroups. However, there exist amenable semigroups admitting no Følner sequences, so we need a different method to prove Theorem 2 ,

Proof of Theorem 2. Let $M \subset P(Y)$ be as before. By Lemma 1, it is a non-empty convex compact subset of $P(Y)$.

Note that the action $\Gamma \curvearrowright Y$ induces an action $\Gamma \curvearrowright P(Y)$ by push-forwards: $\gamma \sigma=\left(S_{\gamma}\right)_{*}(\sigma)$. Also, $\Gamma M \subset M$. Indeed, for any $\gamma \in \Gamma$ and $\sigma \in M$,

$$
\begin{aligned}
p_{*}(\gamma \sigma) & =p_{*}\left(\left(S_{\gamma}\right)_{*}(\sigma)\right)=\left(p \circ S_{\gamma}\right)_{*}(\sigma)=\left(T_{\gamma} \circ p\right)_{*}(\sigma)=\left(T_{\gamma}\right)_{*}\left(p_{*}(\sigma)\right) \\
& =\left(T_{\gamma}\right)_{*}(\mu)=\mu .
\end{aligned}
$$

Hence by Day's fixed-point theorem Day there exists $\nu \in M$ with $\nu=$ $\left(S_{\gamma}\right)_{*}(\nu)$ for all $\gamma \in \Gamma$.

Acknowledgements. The author is grateful to Feliks Przytycki, Marcin Sabok and Gabor Elek for helpful comments. Also thanks are due to the anonymous referee for corrections and suggestions which improved the exposition of the results. 
This research was partially supported by the NCN (National Science Centre, Poland) through the grant Harmonia no. 2018/30/M/ST1/00668.

\section{References}

[Day] M. M. Day, Fixed point theorems for compact convex sets, Illinois J. Math. 5 (1961), $585-596$.

[Kec] A. S. Kechris, Classical Descriptive Set Theory, Grad. Texts in Math. 156, Springer, New York, 1995.

[Pat] A. L. T. Paterson, Amenability, Math. Surveys Monogr. 29, Amer. Math. Soc., 1988.

[Prz] F. Przytycki, Thermodynamic formalism methods in one-dimensional real and complex dynamics, in: Proc. Int. Congress Math. (Rio de Janeiro, 2018), Vol. 3, 21052132.

[Sin] Ya. G. Sinai (ed.), Dynamical Systems II: Ergodic Theory with Applications to Dynamical Systems and Statistical Mechanics, Encyclopaedia Math. Sci. 2, Springer, Berlin, 1989.

Tomasz Cieśla

Department of Mathematics and Statistics

McGill University

805, Sherbrooke Street West

Montreal, Quebec, Canada H3A 2K6

E-mail: tomasz.ciesla@mail.mcgill.ca 